\title{
Spotlight on left ventricular dyssynchrony: Part 3: Diagnostic value and therapeutic guidance
}

\author{
Saurabh Malhotra, MD MPH FASNC, ${ }^{a}$ and Ami E. Iskandrian, MD MASNC ${ }^{b}$ \\ a Division of Cardiology, John H Stroger Hospital of Cook County, Chicago \\ b University of Alabama at Birmingham, Birmingham
}

Received Apr 16, 2021; accepted Apr 16, 2021

doi: $10.1007 / \mathrm{s} 12350-021-02650-6$

Temporal differences in activation and contraction of various myocardial segments of the left ventricle (LV), or LV dyssynchrony (LVD), have been shown to be a prognostic indicator of mortality and malignant arrhythmias among those with heart failure (HF) and reduced ejection fraction. In this regard, it may be useful in identification of HF patients who are unlikely to derive a benefit from a primary prevention implantable cardioverter defibrillator (ICD). In HF patients, additional mortality benefit is derived from effective cardiac resynchronization therapy (CRT). Phase analysis of gated SPECT provides a guidance criterion to improve CRT response, which includes presence of systolic LVD, scar burden, and concordance of left ventricular lead with the site of latest myocardial activation.

Wang et al, in this issue of the Journal, report the value of both systolic and diastolic LVD for predicting CRT response from 84 patients with dilated cardiomyopathy and left bundle branch block (LBBB). Systolic and diastolic LVD were assessed by phase analysis of gated SPECT, with identification of the latest contracting and relaxing segments based on the systolic and diastolic phase polar maps. Over an average follow-up of 36 months, approximately, $60 \%$ of the patients were identified to be CRT responders, based on an improvement in LV ejection fraction $(\mathrm{EF})$ of $\geq 5 \%$. In a multivariate model, systolic and diastolic phase standard deviation (PSD) and histogram bandwidth (HBW) were independent predictors of CRT response. In addition, among patients in whom the LV lead was positioned in the first three latest contracting or relaxing segments (n $=18)$, CRT response was noted in 17 (94\%). In contrast,

Reprint requests: Saurabh Malhotra, MD MPH FASNC, Division of Cardiology, John H Stroger Hospital of Cook County, Chicago; saurabh.malhotra@cookcountyhhs.org

J Nucl Cardiol 2021;28:1021-2.

$1071-3581 / \$ 34.00$

Copyright (C) 2021 American Society of Nuclear Cardiology. among those in whom the LV lead position was in a region remote from the three latest contracting and relaxing segments, CRT response was only noted in 1 out of $15(7 \%)$ patients. This study strengthens the existing data on the need for LV lead concordance with site of latest myocardial contraction or activation, for CRT response, while identifying diastolic LVD and regions of latest relaxation as new variables that can potential further enhanced guidance for LV lead placement.

While application of LVD in HF populations is intuitive, given the high prevalence of abnormal myocardial contractility in HF patients, its utility in diagnosis of coronary artery disease is seldom considered. There is prior evidence that suggests a value of LVD for diagnosis of multivessel CAD, but its role in diagnosing subclinical atherosclerosis or microvascular disease is less studied.

Van Tosh and colleagues, in a gated Rubidium- 82 PET perfusion study, report the association of LVD with myocardial blood flow, among those with and without anatomically significant CAD. Among patients with apparently normal coronary arteries (SSS $<4$, SRS $<4$ and $\%$ stenosis $<70 \%$ ), those with abnormally low myocardial flow reserve $\left(<2 \mathrm{~mL} \cdot \mathrm{g}^{-1} \cdot \mathrm{min}^{-1}\right)$ had more severe LVD as assessed by HBW, when compared to those with MFR $\geq 2 \mathrm{~mL} \cdot \mathrm{g}^{-1} \cdot \mathrm{min}^{-1}$. Among apparently normal arteries with 20\%-69\% stenosis, rest and stress myocardial blood flow (MBF) was lower when compared to arteries with $<20 \%$ stenosis. This correlated with a higher regional HBW among areas supplied by arteries with 20\%-69\% stenosis when compared to apparently normal arteries with $<20 \%$ stenosis. In contrast, patients with significant epicardial coronary artery disease ( $>70 \%$ luminal stenosis) had a much lower rest/stress MBF and MFR, correlated with greater LVD.

Peix and colleagues, in another report published in this issue, assessed LVD on gated rest and gated stress 
SPECT MPI among 218 patients with chest pain, but normal epicardial coronary arteries on angiogram. A proportion of these patients (25\%) had abnormal SPECT MPI defined by SDS $>4$, while the remainder had a normal study. Patient with abnormal SPECT MPI, but normal epicardial coronary arteries had a significant increase in post-stress LVD, measured by both PSD and HBW. These observational studies shed some light on the relationship of LVD with subclinical atherosclerosis, and possibly microvascular dysfunction, in symptomatic patients without significant overt CAD. Incorporation of LVD as a diagnostic marker of subclinical CAD will definitely require further rigorous analysis, and if proven it could serve as one of the earliest markers in the ischemic cascade. Additionally, it remains to be seen if LVD can provide similar diagnostic value as conferred by assessment of MBF, which can be of particular importance in SPECT MPI given the lack of robust MBF assessment with SPECT.

\section{Disclosures}

Dr. Malhotra serves on the speaker's bureau of Pfizer and Alnylam, and on the advisory board of Alnylam. Dr. Iskandrian has nothing to disclose.

Publisher's Note Springer Nature remains neutral with regard to jurisdictional claims in published maps and institutional affiliations. 